\title{
Percepción de seguridad sanitaria del consumidor quiteño en el servicio de transporte aéreo post COVID-19
}

\section{Perception of Sanitary Safety of Quito's consumer in the Post- COVID-19 Air Transport Service}

Sebastián Carvajal Alcívar¹. Luisa Flores Núñez ${ }^{2 *}$, Almudena Mayorga ${ }^{3}$, Freddy Xavier Lasso Garzón ${ }^{4}$

${ }^{1}$ Universidad de las Américas, Escuela de Hospitalidad y Turismo. Av. de los Granados E12-41 y Colimes esq. 170503.

Quito, Ecuador.

$凶$ sebastian.carvajal@udlanet.ec

https://orcid.org/0000-0003-0112-1579

${ }^{2}$ Universidad de las Américas, Escuela de Hospitalidad y Turismo. Av. de los Granados E12-41 y Colimes esq. 170503. Quito, Ecuador.

\luisa.flores@udla.edu.ec

https://orcid.org/0000-0002-0527-9755

${ }^{3}$ Universidad de las Américas, Escuela de Hospitalidad y Turismo. Av. de los Granados E12-41 y Colimes esq. 170503. Quito, Ecuador.

凶almudena.mayorga@udla.edu.ec

https://orcid.org/0000-0002-1377-8984

${ }^{4}$ Universidad de las Américas, Escuela de Hospitalidad y Turismo. Av. de los Granados E12-41 y Colimes esq. 170503.

Quito, Ecuador.

$凶$ freddy.lasso@udla.edu.ec

https://orcid.org/0000-0002-5484-4562

\begin{abstract}
Resumen
La aviación es una actividad que ha ido desarrollándose a lo largo del tiempo, adaptándose a las necesidades que surgen en los consumidores, ya que este es el principal medio de transporte para la realización del turismo. Así mismo, el turismo es una importante fuente de ingresos para muchos países; la cual se ha visto drásticamente afectada de manera negativa por la pandemia de la COVID-19, actividad que involucra establecimientos turísticos como hoteles, agencias de viajes, restaurantes, y del que se analizará en el presente artículo: el servicio de transporte aéreo. Por consiguiente, el objetivo principal de esta investigación es analizar la percepción de seguridad sanitaria del consumidor quiteño en el servicio de transporte aéreo post COVID-19, y así lograr determinar los diferentes tipos de perfiles con características determinadas de los consumidores de este servicio, utilizando una metodología con enfoque cualitativo, aplicando encuestas estructuradas a los ciudadanos quiteños mayores a dieciocho años. Con la información obtenida, se procedió a una tabulación, escogiendo los resultados de cinco preguntas que se consideraron de mayor relevancia, así como también se realizaron cuatro correlaciones con los datos de seis preguntas tomando en cuenta diferentes variables. Los resultados arrojaron tres perfiles de diferentes rangos de edad y características en cuanto a preferencias de consumo del servicio.
\end{abstract}

Palabras clave: aeropuerto, coronavirus, turismo, bioseguridad, pasajero. 


\begin{abstract}
Aviation is an activity that has been developed in the last decades, adapting to the needs that arise in consumers, since this is the main means of transport for tourism. Likewise, tourism is an important source of income for many countries; which has been drastically affected in a negative way by the Covid-19 pandemic, an activity that involves tourist establishments such as hotels, travel agencies, restaurants and air transport, that will be analyzed in this article. Therefore, the main objective of this research is to analyze the perception of health security of the Quito consumer in the post Covid-19 air transport service and thus, to determine the different types of profiles with certain characteristics of the consumers of this service, using a methodology with a qualitative approach, applying structured surveys to Quito citizens over eighteen years of age. With the information obtained, a tabulation was made, choosing the results of five questions that were considered of greater relevance, as well as four correlations with the data of six questions taking into account different variables. The results yielded three profiles of different age ranges and characteristics in terms of service consumption preferences.
\end{abstract}

Keywords: airport, Coronavirus, tourism, biosecurity, passenger.

\section{Introducción}

Actualmente, el planeta vive una situación muy difícil, la pandemia causada por el virus COVID-19 ha provocado muchísimas complicaciones a nivel mundial, no únicamente en el área de la salud, sino que ha afectado de manera abrupta a diversos sectores sociales y económicos, entre ellos, la aviación. La COVID-19, es un nuevo tipo de coronavirus que afecta la salud de las personas, se transmite de una persona a otra por contacto directo, indirecto o cercano con personas infectadas por medio de secreciones corporales (Organización Mundial de la Salud [OMS], 2020a).

La aviación es una actividad vulnerable a diversos factores externos, como el caso de una pandemia. Anteriormente, la aviación ya había pasado por una crítica situación con el brote de la enfermedad del SARS en 2003, la cual, según la Asociación Internacional de Transporte Aéreo (IATA por sus siglas en inglés), provocó una reducción del $45 \%$ de la demanda de vuelos en Asia, por lo que cabe decir que la COVID-19 ha tenido un mayor impacto en esta actividad, ya que surgió a nivel mundial (Suau-Sanchez et al., 2020).

En el contexto de la pandemia, la aviación ha sido uno de los principales medios de dispersión del virus, especialmente en aeropuertos y aviones, los cuales han sido grandes focos de contagio, lo que ha provocado una serie de consecuencias económicas en la industria de la aviación y secuelas psicológicas en los consumidores del transporte aéreo. Todas las aerolíneas a nivel mundial se han visto afectadas. La IATA hizo público su análisis financiero, según el cual se espera que las aerolíneas pierdan $\$ 84,3$ mil millones de dólares en 2020. Se estima que los ingresos caerán un $50 \%$ a $\$ 419$ mil millones de $\$ 838$ mil millones en 2019 (Larenas, 2020).

El efecto de la pandemia a nivel social ha sido devastador, solamente entre abril y junio del presente año, se registró una disminución de la cantidad de horas de trabajo a escala mundial del $14 \%$, equiparable a 400 millones de empleos a tiempo completo, los países de ingresos medianos y bajos son los más afectados, pues su disminución ha sido del 16,1\% (Organización Internacional del Trabajo [OIT], 2020). Estas consecuencias han sido el resultado del cierre de fronteras para disminuir la dispersión del virus, la cuarentena impuesta a la población mundial y diversas medidas que han tomado la mayoría de países del mundo.

Aun así, el principal problema que enfrentan hoy en día las aerolíneas y aeropuertos en todo el mundo es el cambio de perspectiva por parte del consumidor. La industria de la aviación se encuentra muy afectada por la pandemia y se debe a la falta de consumidores, que sufre actualmente este sector. El consumidor del transporte aéreo, a raíz de la pandemia ha cambiado sus prioridades y su posición con respecto a las necesidades que tiene cuando utiliza este servicio.

Siendo la aviación el principal medio de transporte de turismo receptivo en Ecuador, este sector económico también se ha visto afectado por la poca o nula llegada de turistas extranjeros al país. El aeropuerto de la capital, en el segundo trimestre del año 2019, registró un total de 
294.404 turistas que ingresaron por vía aérea al país, pero en el mismo período del 2020 este número se redujo drásticamente en un $98,58 \%$ pues el total de turistas fue de 4.172 , debido al cierre de operaciones a causa de la pandemia (Ministerio de Turismo [MINTUR], 2020a). El sector turístico ha registrado pérdidas de hasta 540 millones de dólares (López, 2020). Por ello, el Gobierno ecuatoriano busca la reactivación del turismo de manera paulatina; para ello, como primer paso, el Ministerio del Turismo recibió el sello Safe Travels que otorga el Consejo Mundial de Viajes y Turismo [WTTC], el cual es otorgado a destinos globales que cumplen protocolos de bioseguridad estandarizados en los establecimientos turísticos para, de esta manera, poder recuperar la confianza de los viajeros (MINTUR, 2020b).

Sin embargo, existen planes a nivel mundial que impulsan la reactivación del transporte aéreo por medio de distintas medidas de bioseguridad y distanciamiento social para la pronta apertura de la aviación. Por ejemplo, en Ecuador, en junio del 2020 fueron reaperturadas las operaciones aéreas en el país, pero la recuperación de este sector está ligada y muy marcada por las restricciones de bioseguridad dictadas por las autoridades ecuatorianas, tal como la prueba PCR (prueba de proteína $\mathrm{C}$ reactiva) para detectar el virus, así como el aislamiento preventivo obligatorio (APO) (aunque esta medida se eliminó, desde el 15 de agosto, para aquellos viajeros que ingresen al país con una prueba PCR negativa); también se encuentra limitada por las restricciones propias de cada país. Además, el presidente de la Asociación de Aerolíneas de Ecuador, Marcos Subía, indicó que, a nivel de América Latina, puede que en 3 años se logre alcanzar la regularidad con la que operaba esta industria en 2019 (Subía, 2020).

Tras haber analizado la situación actual de estas industrias - tanto el turismo como la aviación en el Ecuador-, se llevó a cabo esta investigación con el objetivo de conocer la percepción del consumidor quiteño, profundizar acerca de las necesidades de este en la etapa pospandemia $\mathrm{y}$, con el análisis de los resultados, crear distintos perfiles de los consumidores del transporte aéreo basados en la edad. Se realizó este estudio porque no se han efectuado estudios del comportamiento del consumidor del transporte aéreo de la ciudad de Quito en el pasado, y se consideró esta situación como una oportunidad para entender de mejor manera las necesidades de este segmento.
Esta investigación se basó en la aplicación de encuestas a usuarios del servicio de este tipo de transporte, se tabularon los datos obtenidos y se obtuvo respuestas relevantes; después de realizar un análisis estadístico de las preguntas de manera individual y de correlaciones entre algunas de ellas, se crearon 3 perfiles en los que se detalla las preferencias de los consumidores dependiendo de la edad que estos tengan.

\section{Materiales y Métodos}

La investigación propuesta se desarrolló considerando la percepción del viajero aéreo con relación a la seguridad sanitaria que ofrece este servicio. Esto permitió la asimilación real de los efectos que ha producido la pandemia en el comportamiento del consumidor del servicio de transporte aéreo en la ciudad de Quito. Para esto, se consideró un enfoque de carácter cuantitativo y se tomó en cuenta a los residentes de la ciudad de 18 años en adelante. Según el Sistema Nacional de Información [SIN] en 2020 son 1.800 .009 las personas que pertenecen a este segmento; esta cifra es una aproximación (SNI, 2017). Se tomó a este grupo etario debido a que a partir de los18 años las personas pertenecen a la población económicamente activa del país. Para obtener una muestra de esta población se aplicó la fórmula para poblaciones finitas [Ecuación 1], tomada del libro Estadística y muestreo (Martínez Bencardino, 2012).

$$
n=\frac{N z^{2} p q}{(N-1) * E^{2}+z^{2} p q}
$$

donde:

- $\quad \mathrm{N}=$ población total $=1.800 .009$

- $\quad \mathrm{z}=$ nivel de confianza $=1,96(95 \%)$

- $\mathrm{p}=$ probabilidad de que ocurra el evento estudiado $=0,5$

- $\quad \mathrm{q}=$ probabilidad de que NO ocurra el evento estudiado $=0,5$

- $\quad \mathrm{E}=$ margen de error $=0,05(5 \%)$

Como resultado de la fórmula se obtuvo un resultado de 388 , que corresponde al número de personas a las que se les aplicó una encuesta estructurada, compuesta de 20 preguntas cerradas relacionadas a la seguridad sanitaria en el servicio de transporte aéreo. Para formular las preguntas, se 
tomó en cuenta todos los elementos involucrados en este servicio, tales como aeropuertos, aerolíneas, aviones y las nuevas medidas adoptadas por cada uno de ellos. Las preguntas fueron planteadas por los autores de la investigación y las encuestas fueron respondidas de manera virtual por viajeros que hacen uso del transporte aéreo de una manera frecuente. El cuestionario se efectuó durante los primeros meses de la pandemia de COVID-19 en el Ecuador (mayo) debido al inesperado cambio de perspectiva en la mente del consumidor del transporte aéreo. Este estudio no se realizó de manera presencial debido a las restricciones de movilidad que dispuso el COE nacional durante los primeros meses de la emergencia sanitaria.

Al finalizar este proceso, se procedió a tabular los datos obtenidos de las encuestas y se escogieron los resultados de 5 preguntas que resultaron ser de mayor significación para interpretarlos. Estas preguntas fueron elegidas por la relevancia y trascendencia de sus respuestas, y se consideraron en el análisis y en los resultados obtenidos. Por otro lado, no se tomó en cuenta a las preguntas restantes debido a que las respuestas, en su mayoría, coincidían.

Además, para este estudio se empleó la fórmula de chi-cuadrado $\left(\chi^{2}\right)$ que determina el estado de dependencia e independencia de la variables cualitativas mediante el análisis de los resultados; también se utilizó la correlación de Spearman, esta permite determinar la fortaleza de la asociación entre dos variables aleatorias y se puede conocer si la relación es producto del azar o de un patrón; se realizaron cuatro correlaciones con 6 preguntas debido a que solamente en estas existió un estado de dependencia, y se procedió a interpretar los resultados obtenidos. En cada correlación se planteó dos hipótesis, con la ayuda del coeficiente de correlación de Spearman se pudo concluir si las variables son dependientes o independientes, pues se conoce que sus valores oscilan entre $-1 \mathrm{y}+1$, cuanto más cercano a uno, existirá mayor correlación entre las variables y esta será directamente proporcional; cuanto más cercano a cero la correlación será más baja (Hernández Sampieri et al., 2013). Para las correlaciones se utilizó el software SPSS [Statistical Product and Service Solutions], este permite hacer tratamientos sistemáticos de las variables, elaborar cuadros, obtener tablas de contingencia y gráficos según las necesidades de la investigación (Meulman \& Heiser, 2005).

\section{Resultados}

Una vez realizada la encuesta se obtuvieron distintos datos importantes sobre el impacto que ha tenido la pandemia de COVID-19 en el consumidor quiteño. Se tomaron en cuenta algunos de los datos más relevantes, específicamente de 5 preguntas, que se presentan a continuación:

- Pregunta 7: ¿Qué aspectos consideraría más peligrosos al momento de consumir servicios de transporte aéreo, post Covid19?

- Pregunta 10. ¿Cree usted que las aerolíneas deberían pedir información acerca del estado de salud del pasajero antes de la compra de un boleto aéreo?

- $\quad$ Pregunta 17. Puede que estas nuevas medidas en el servicio de transporte aéreo consuman más tiempo de lo regular, ¿qué tan probable es que tolere el tiempo de espera?

- Pregunta 19. ¿Qué medidas considera adecuadas para poder viajar tranquilo después de la pandemia (COVID-19)?

- Pregunta 20: Si en un futuro existe una vacuna para prevenir la COVID 19, ¿cree usted que esta debería ser obligatoria para el embarque en el transporte aéreo?

El aspecto que se considera más peligroso, con un $36,82 \%$, es la "proximidad con otros consumidores" pues se sabe que muchos contagiados son asintomáticos y al tener contacto con varias personas hace que los pasajeros se sientan inseguros al viajar. Por otro lado, el segundo factor considerado más peligroso para el consumidor, con un $30,34 \%$, es la "falta de higiene por parte de los demás consumidores", tal cual se muestra en la Figura 1. El resultado del análisis se interpreta alrededor del bajo nivel de confianza que tiene el consumidor hacia otros pasajeros desconocidos, pues no todos los pasajeros cumplen a cabalidad con las medidas de bioseguridad impuestas por la OMS. Se sabe que el virus se transmite por medio de gotículas que expulsan la nariz y la boca, aun así, estas no tienen un largo alcance por su peso (OMS, 2020a); por lo tanto, el estar próximo a otros consumidores aumenta el riesgo de contagio y la falta de higiene de los mismos puede ocasionar un contacto directo con el virus. 


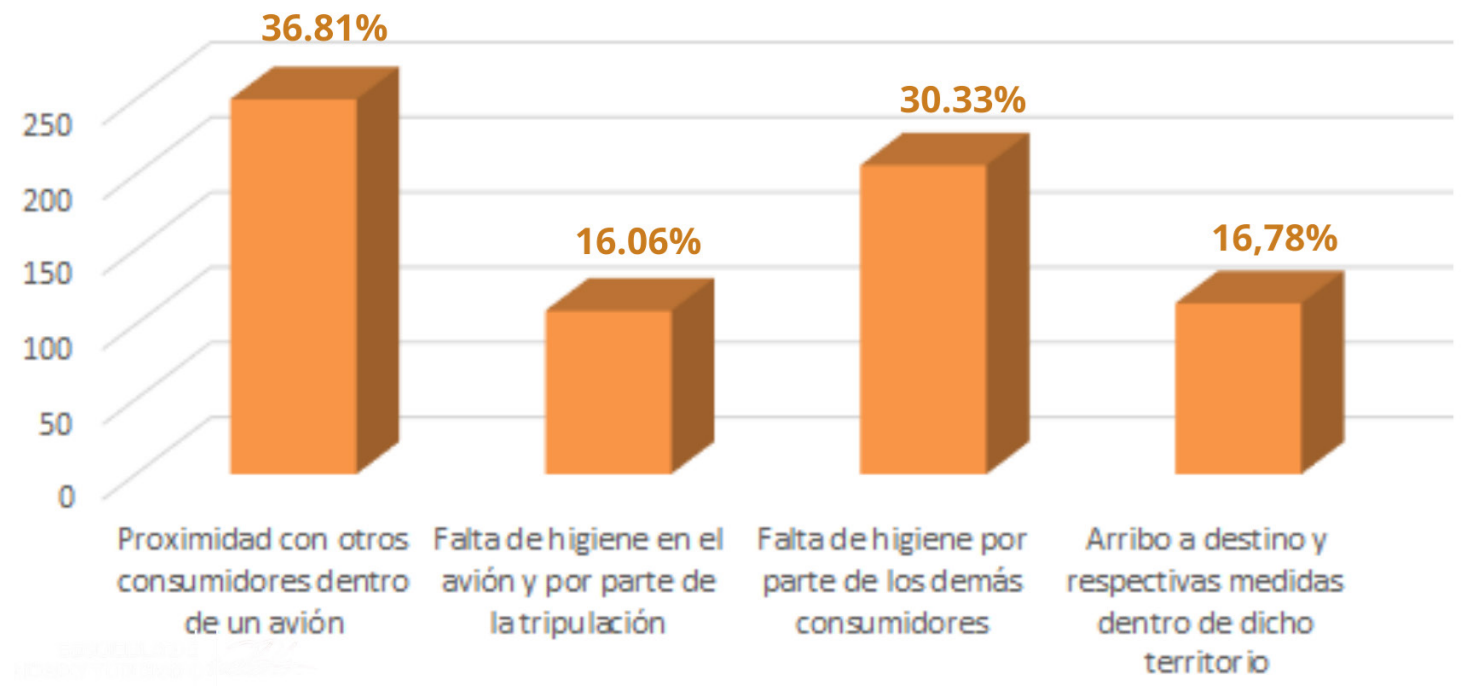

Figura 1. Aspectos considerados más peligrosos al momento de consumir servicios de transporte aéreo, post COVID-19

El 95,36\% de los encuestados están de acuerdo en que las aerolíneas deberían pedir información acerca del estado de salud del pasajero antes de la compra de un boleto aéreo, pues consideran que es una medida importante para sentirse seguros durante su viaje, mientras que el 4,6\% restante está en desacuerdo con esta medida, tal como se muestra en la Figura 2, porque consideran innecesario revelar su estado de salud por conceptos de confidencialidad, intimidad e, incluso, porque pueden llegar a sentir vergüenza. La mayoría de los encuestados coinciden en que las aerolíneas deberían pedir información acerca del estado de salud del viajero; esta medida ha sido adoptada por varias aerolíneas, un ejemplo es IBERIA, que como requerimiento de las autoridades, necesita que el pasajero acepte una declaración de salud durante el check-in de su vuelo para confirmar que: no tiene síntomas, no ha sido diagnosticado con COVID-19, no ha estado en contacto directo con una personas que lo padeciera y no ha estado en periodo de cuarentena obligatoria (IBERIA, 2020).

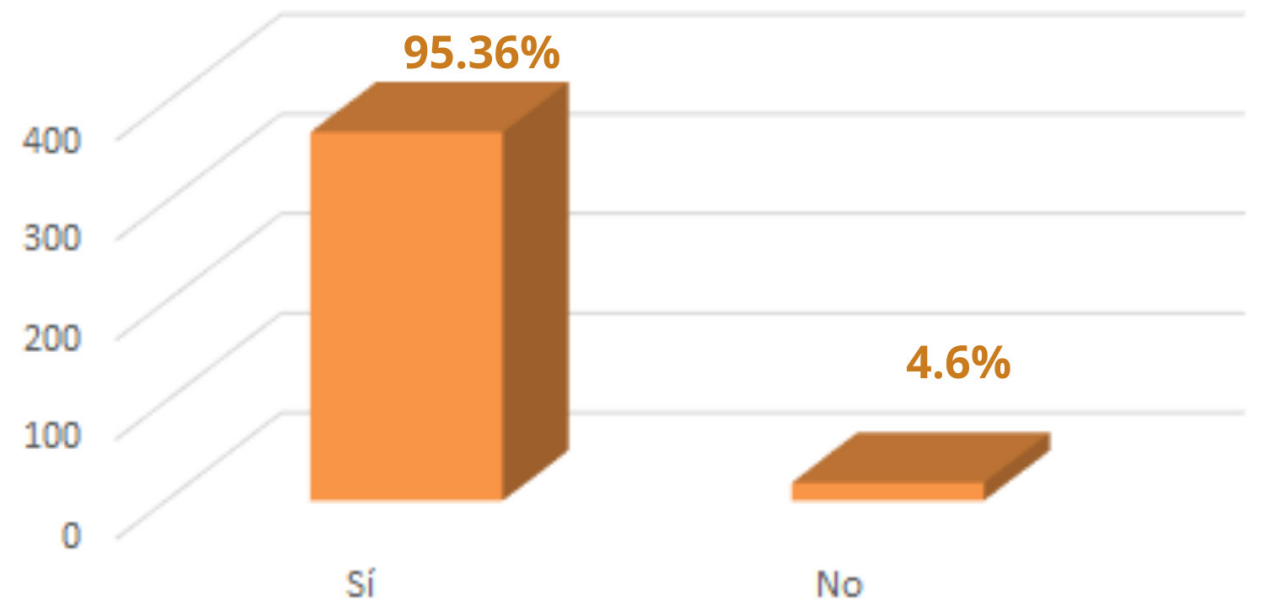

Figura 2. Solicitud por parte de las aerolíneas de información acerca del estado de salud del pasajero antes de la compra de un boleto aéreo 
El 40,20\% de los encuestados considera que es muy probable tolerar el tiempo de espera de las posibles nuevas medidas en los aeropuertos, el 47,65\% lo considera probable pero no se encuentran totalmente convencidos, el 8,76\% puede llegar a tolerarlo muy poco, el $1,80 \%$ de los encuestados no toleran por ningún motivo más tiempo de espera por las nuevas medidas y al $1,54 \%$ le es indiferente el tiempo de espera, como se muestra en la Figura 3. El cambio en la psicología de las personas se encuentra adherido a la constante información sobre los impactos de la pandemia, que reciben cada día a través de distintos medios sociales y digitales. La exposición excesiva a este tipo de información y noticias causa que el miedo al contagio sea mayor. Según el escritor científico David Robson (2020), este miedo hace que las personas sean más conformistas y menos receptivos a la excentricidad (como se citó en Whitehead, 2020); logrando así que los viajeros toleren de mejor manera el tiempo de espera en el aeropuerto.

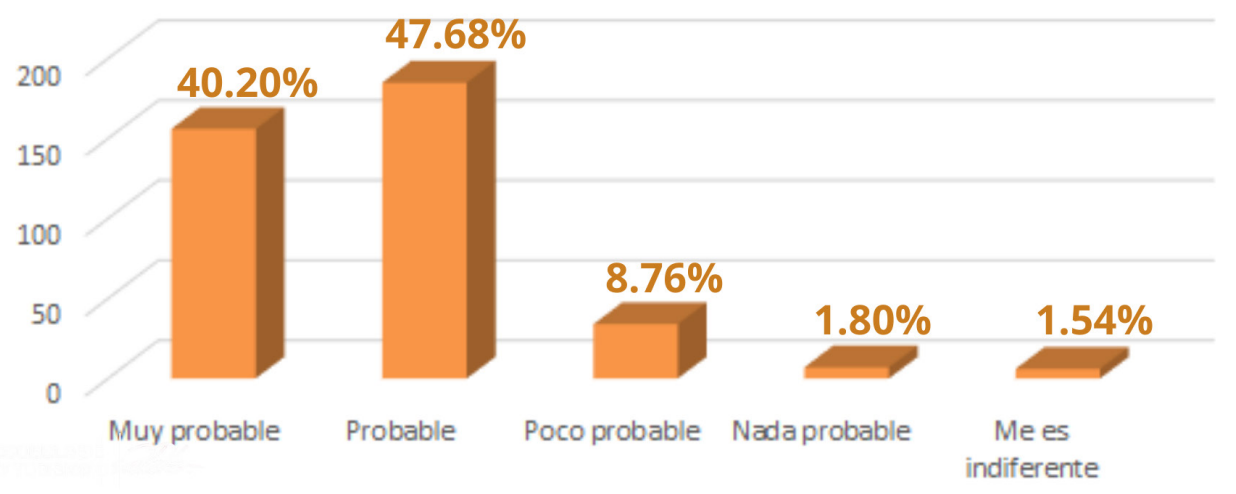

Figura 3. Tolerancia a un mayor tiempo de espera por las nuevas medidas en el servicio de transporte aéreo

En relación con las medidas para un viaje tranquilo, la respuesta más votada fue "nuevos protocolos de sanidad y seguridad en las aerolíneas" con un $25,84 \%$, pues el consumidor considera que las aerolíneas deberían tomar las medidas necesarias para que los clientes logren sentirse cómodos y seguros en su viaje aéreo. En segundo lugar, con un $21,57 \%$ está "dejar un asiento vacío entre los pasajeros", pues de esta manera se cumpliría con la medida de distanciamiento físico y con el 15,19\% "medición de temperatura antes de abordar y al bajar del avión', como lo detalla la Figura 4, pues es una herramienta con la que se puede detectar síntomas del virus de una manera más rápida. Todas estas medidas han sido adoptadas por varios aeropuertos a nivel mundial; adaptándose a los nuevos comportamientos y preferencias de los viajeros, el aeropuerto internacional de Quito no ha sido la excepción, puesto que desde el mes de junio del año 2020 dispuso distintos protocolos dictados por el COE nacional que aseguran el bienestar del viajero y empleados, además de disminuir la probabilidad del contagio y dispersión del virus (Aeropuerto Internacional de Quito, 2020).

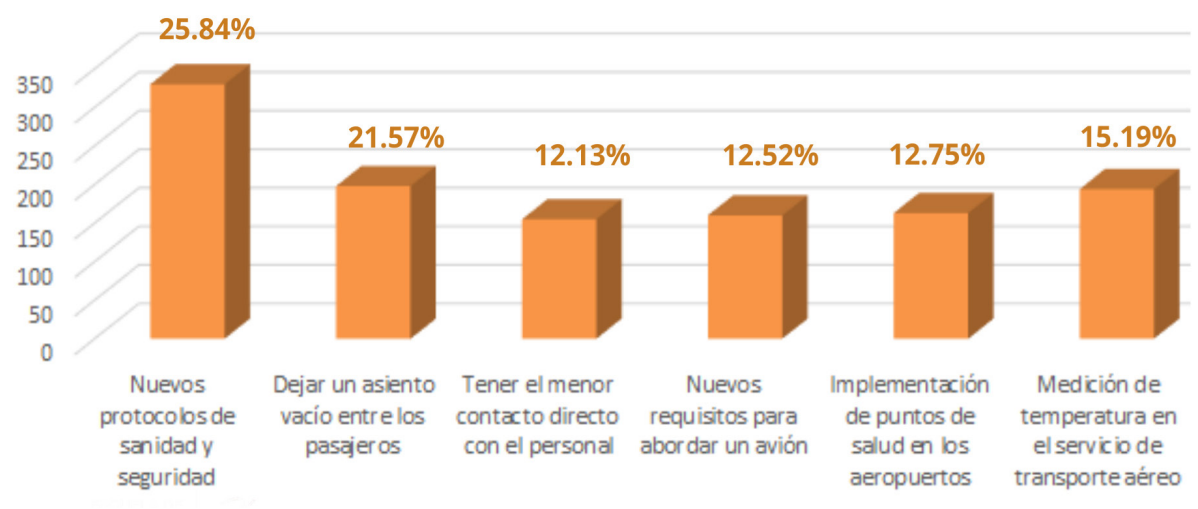

Figura 4. Medidas adecuadas para poder viajar tranquilo después de la pandemia (COVID-19) 
El $87,88 \%$ de los encuestados opinan que la vacuna debe ser obligatoria para el embarque en el transporte aéreo, mientras que el $12,11 \%$ opina que esta medida no debería ser obligatoria, como se muestra en la Figura 5, porque muchas personas no están de acuerdo con el uso de vacunas y se muestran reacias ante esta medida sanitaria. En su mayoría las personas encuestadas creen que la vacuna debe ser un requisito para el embarque en una terminal aérea, pues según afirma la Organización Mundial de la Salud (OMS), la vacunación es de gran importancia, debido a que causa una inmunización de determinados virus en el entorno, además la vacuna es una forma segura y eficaz de prevenir enfermedades y salvar vidas (OMS, 2020b). Por otro lado, la minoría no está a favor de esta medida, pues el proceso de elaboración de una vacuna es complejo y tarda al menos entre 10 y 15 años, puesto que esta debe pasar por distintas etapas como: exploración (2 a 4 años), preclínica (1 a 2 años), solicitud para investigar un medicamento nuevo (30 días); una vez que se ha aprobado la solicitud, comienzan las 4 fases de ensayos en personas, posterior a los ensayos empieza el análisis de efectos adversos de la vacuna $\mathrm{y}$, finalmente, cuando una vacuna aprueba todas las fases previas se procede a su comercialización (The College of Physicians of Philadelphia, 2018). Es por este motivo que la vacunación contra la COVID-19 no es confiable para cierto número de personas, ya que las vacunas actuales se encuentran desarrollándose de manera acelerada y la población no encuentra a la vacuna como fidedigna, y esta puede tener efectos adversos por la rapidez de los ensayos en personas.

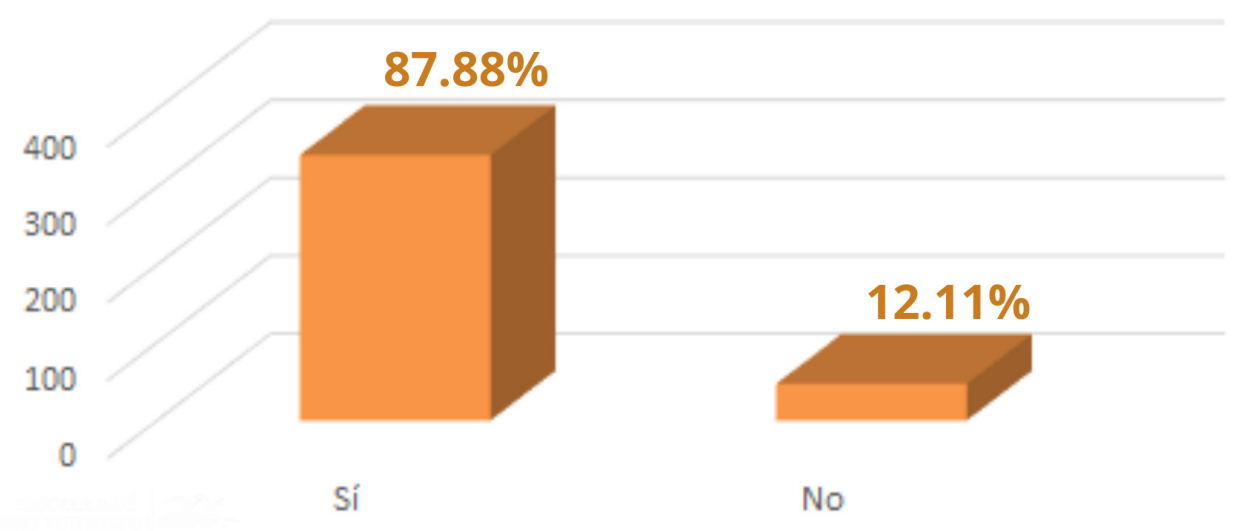

Figura 5. Si en un futuro existe una vacuna para prevenir el COVID 19, ¿cree usted que esta debería ser obligatoria para el embarque en el transporte aéreo?

Se realizaron cuatro correlaciones con los datos de 6 preguntas. Para la primera correlación se tomaron en cuenta las siguientes variables:

- Pregunta 2: Edad de los encuestados

- $\quad$ Pregunta 6: ¿Qué tan probable es que viaje en avión en la etapa post COVID-19? Califique en la escala siendo 5 muy probable y 1 nada probable.

Se plantearon 2 hipótesis, una nula y una alternativa:

- $\quad \mathrm{H}_{0}$ : La probabilidad de viajar en avión es independiente a la edad.

- $\quad \mathrm{H}_{1}$ : La probabilidad de viajar en avión es dependiente de la edad.
La significación de la prueba de chi-cuadrado de Pearson $(0,000)$ (Tabla 1$)$ y del coeficiente de correlación de Sperman $(0,091)$ (Tabla 2), toma valores inferiores al nivel de significación del 0,05 considerado en este estudio. Esto indica que el estado de las variables analizadas es dependiente y la correlación entre ellas es débil. Entonces, se acepta $\mathrm{H}_{1}$ y existe una dependencia en la probabilidad de viaje y la edad, por lo tanto, los resultados arrojan que es más probable que el 65,21\% de las personas mayores de 60 años viajen en la etapa post COVID-19; mientras que $51,38 \%$ de las personas con edades entre 36 y 60 años es nada probable que decidan viajar en avión en la etapa post COVID-19. Para la obtención de estos porcentajes se realizó una sumatoria de los resultados obtenidos de la Figura 6. Por lo que, se interpreta que existen distintos factores que se in- 
volucran en la probabilidad de viaje de las personas mayores de 60 años, puesto que, conforme a un estudio realizado por la Universidad Nacional de Quilmes se determinó que los adultos mayores son un segmento atractivo de consumidores de turismo, ya que son un grupo en crecimiento, con disponibilidad de tiempo e ingresos monetarios por su jubilación; estos elementos en conjunto hacen que este grupo etario se convierta en el más atractivo para reactivar el turismo después de la pandemia (Murello, 2017). Estos pueden ser: mayor disponibilidad de tiempo y mayores ingresos. Es menos probable que las personas con un rango de edad entre 36 y 60 años viajen por la poca disponibilidad de tiempo que poseen, sin embargo, este grupo etario puede considerar viajar por motivos de negocios.

Tabla 1. Pruebas de chi-cuadrado - correlación 1

\begin{tabular}{llcc}
\hline & Valor & Df & Significación asintótica (bilateral) \\
\hline Chi-cuadrado de Pearson & $150,566^{\mathrm{a}}$ & 8 & 0.000 \\
\hline
\end{tabular}

Tabla 2. Medidas simétricas - correlación 1

\begin{tabular}{llcccc}
\hline & Valor & $\begin{array}{c}\text { Error estándar } \\
\text { asintótico }\end{array}$ & T aproximada & $\begin{array}{c}\text { Significación } \\
\text { aproximada }\end{array}$ \\
\hline $\begin{array}{l}\text { Intervalo por } \\
\text { intervalo }\end{array}$ & R de Pearson & 0,174 & 0,062 & 3,462 & $0,001^{\mathrm{c}}$ \\
$\begin{array}{l}\text { Ordinal por } \\
\text { ordinal }\end{array}$ & $\begin{array}{l}\text { Correlación de } \\
\text { Spearman }\end{array}$ & 0,091 & 0,058 & 1,786 & $0,075^{\mathrm{c}}$ \\
\hline
\end{tabular}

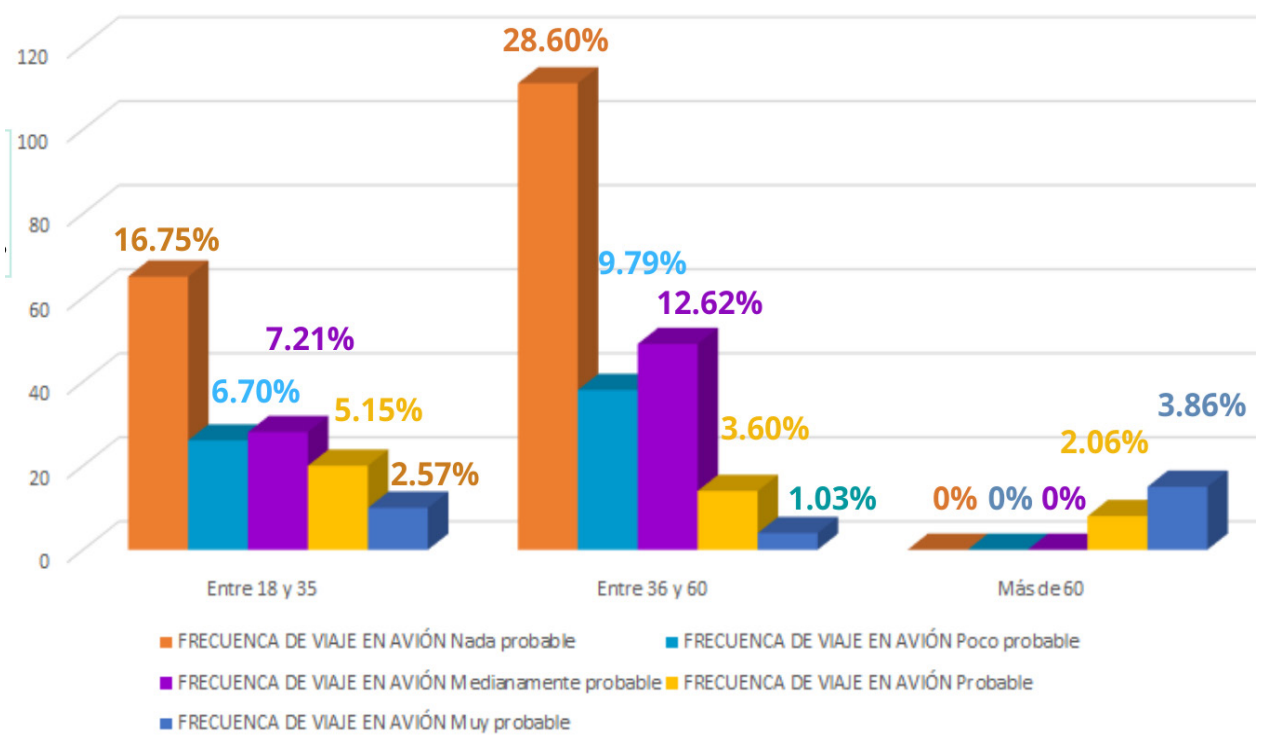

Figura 6. Probabilidad de viaje vs. edad.

Para la segunda correlación se tomó en cuenta las siguientes variables:

- Pregunta 2: Edad de los encuestados.
- Pregunta 11: Tipo de asiento que preferiría utilizar en su próximo viaje aéreo: posición inversa del asiento central de la fila triple, mampara acrílica traslúcida en asientos existentes 
Se plantearon 2 hipótesis, una nula y una alternativa:

- $\quad \mathrm{H}_{0}$ : Las medidas de seguridad adoptadas en el avión son independientes a la edad.

- $\quad \mathrm{H}_{1}$ : Las medidas de seguridad adoptadas en el avión son dependientes de la edad.

La significación de la prueba de chi-cuadrado de Pearson $(0,000)$ (Tabla 3 ) y del coeficiente de correlación de Sperman $(-0,025)$ (Tabla 4), toman valores inferiores al nivel de significación del 0,05 considerado en este estudio. Esto indica que el estado de las variables analizadas es dependiente y la correlación entre ellas es débil. Es así que existe una dependencia en las medidas de seguridad con la edad del pasajero, por lo tanto, los resultados arrojan que las personas entre 18 y 35 estarían de acuerdo con el uso de cualquier de las 2 opciones ya que el uso de la mampara es preferido por el 52,34 \%, mientras que la posición inversa del asiento el 47,65 \%. El $65,27 \%$ de las personas entre 36 y 60 años prefieren el uso de mamparas acrílicas en los asientos existentes y el 78,26\% de las personas mayores a 60 años preferirían el uso de asientos en posición inversa. Para la obtención de estos porcentajes se realizó una sumatoria de los resultados obtenidos de la Figura 7. Este virus se transmite por medio de gotículas que expulsan la nariz y la boca de los infectados (OMS, 2020a) tanto la mampara acrílica como el asiento del medio de la fila triple en posición inversa aseguran que el viajero se encuentre en un volumen aislado que evita la propagación por respiración y reduce la probabilidad de contagio entre los pasajeros (Aviointeriors, 2020). Se interpreta que la preferencia a estos implementos de seguridad depende principalmente de los gustos del consumidor y el nivel de confianza que le pueda ofrecer cualquiera de estas dos opciones.

Tabla 3. Pruebas de chi-cuadrado - correlación 2

\begin{tabular}{llcc}
\hline & Valor & Df & Significación asintótica (bilateral) \\
\hline Chi-cuadrado de Pearson & $19,020^{\mathrm{a}}$ & 2 & 0.000 \\
\hline
\end{tabular}

Tabla 4. Medidas simétricas - correlación 2

\begin{tabular}{llcccc}
\hline & Valor & $\begin{array}{c}\text { Error estándar } \\
\text { asintótico }\end{array}$ & T aproximada & $\begin{array}{c}\text { Significación } \\
\text { aproximada }\end{array}$ \\
\hline $\begin{array}{l}\text { Intervalo por } \\
\text { intervalo }\end{array}$ & R de Pearson & 0,002 & 0,053 & 0,045 & $0,964^{\mathrm{c}}$ \\
$\begin{array}{l}\text { Ordinal por } \\
\text { ordinal }\end{array}$ & $\begin{array}{l}\text { Correlación de } \\
\text { Spearman }\end{array}$ & $-0,025$ & 0,053 & $-0,495$ & $0,621^{\mathrm{c}}$ \\
\hline
\end{tabular}

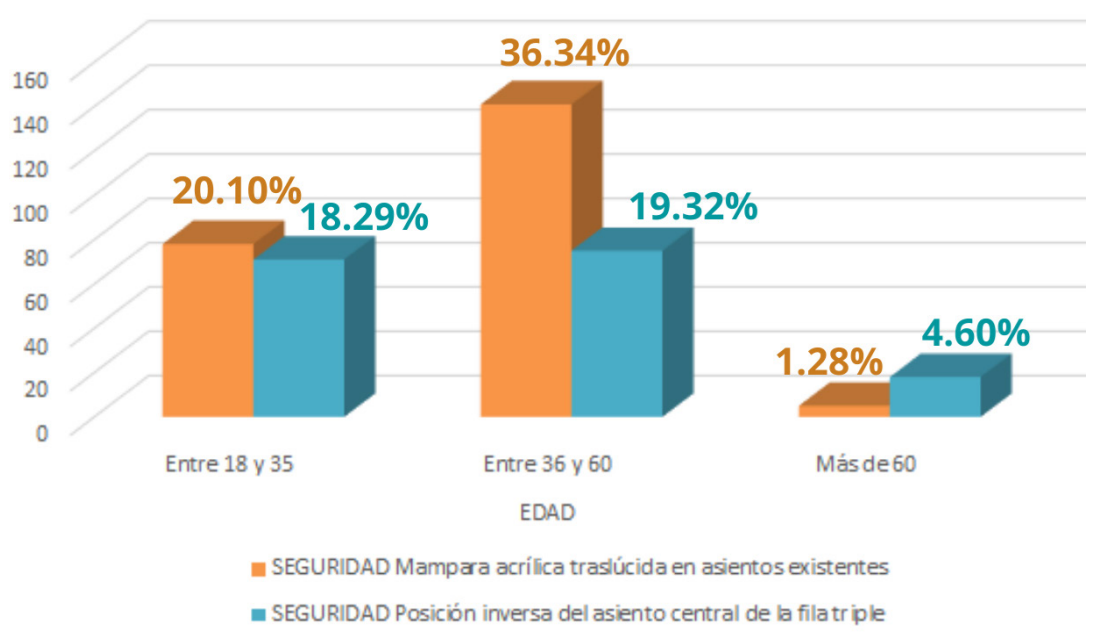

Figura 7. Implementos de seguridad vs. edad. 
Para la tercera correlación se tomó en cuenta 2 variables:

- $\quad$ Pregunta 6: ¿Qué tan probable es que viaje en avión en la etapa post COVID-19? Califique en la escala siendo 5 muy probable y 1 nada probable.

- $\quad$ Pregunta 14: ¿Cree usted necesario que en la etapa post COVID-19 los aeropuertos deberían reducir el número de vuelos por día (arribos y salidas) con el objetivo de evitar la aglomeración de personas?

Se plantearon 2 hipótesis, una nula y una alternativa:

- $\quad \mathrm{H}_{0}$ : La reducción de pasajeros durante los vuelos en la etapa post COVID-19 es independiente a la probabilidad de viaje de las personas.

- $\quad \mathrm{H}_{1}$ : La reducción de pasajeros durante los vuelos en la etapa post COVID-19 es dependiente de la probabilidad de viaje de las personas.

La significación de la prueba de chi-cuadrado de Pearson $(0,002)$ (Tabla 5) y del coeficiente de correlación de Spearman $(0,189)$ (Tabla 6), toman valores inferiores al nivel de significación del 0,05 considerado en este estudio. Esto indica que el estado de las variables analizadas es dependiente y la correlación entre ellas es débil. Existe una dependencia entre la reducción del número de vuelos por día y la probabilidad de viaje de las personas. Los resultados arrojan que el 84,09\% de las personas que tienen menos probabilidad de viajar están de acuerdo con la medida de reducción del número de vuelos por día, de igual manera existe una inclinación por esta medida en el 55,17\% de las personas que tienen más probabilidad de viajar en la etapa post COVID-19. Para la obtención de estos porcentajes se realizó una sumatoria de los resultados obtenidos de la Figura 8. Según el Ministerio de Salud y Protección Social de Colombia, el viajar en avión no representa un riesgo mayoritario para los viajeros, ya que la probabilidad de contagio en la cabina disminuye por la constante purificación de aire con la que cuentan los aviones, además del cumplimiento de las medidas de bioseguridad; sin embargo, el verdadero riesgo de contagio se encuentra presente en las terminales de los aeropuertos por las constantes aglomeraciones y el incumplimiento de los protocolos de bioseguridad (Ministerio de Salud y Protección Social de Colombia. [MinSalud], 2020). Es por este motivo que la reducción de vuelos por día, genera mayor seguridad en la mayoría de los encuestados debido a que esta medida reduce las aglomeraciones en aeropuertos y la propagación del virus en los mismos.

Tabla 5. Pruebas de chi-cuadrado - correlación 3

\begin{tabular}{lccc}
\hline & Valor & Df & Significación asintótica (bilateral) \\
\hline Chi-cuadrado de Pearson & $17,540^{\mathrm{a}}$ & 4 & 0.002 \\
\hline
\end{tabular}

Tabla 6. Medidas simétricas - correlación 3

\begin{tabular}{|c|c|c|c|c|c|}
\hline & & Valor & $\begin{array}{l}\text { Error estándar } \\
\quad \text { asintótico }\end{array}$ & T aproximada & $\begin{array}{c}\text { Significación } \\
\text { aproximada }\end{array}$ \\
\hline $\begin{array}{l}\text { Intervalo por } \\
\text { intervalo }\end{array}$ & R de Pearson & 0,192 & 0,052 & 3,843 & $0,000^{\mathrm{c}}$ \\
\hline $\begin{array}{l}\text { Ordinal por } \\
\text { ordinal }\end{array}$ & $\begin{array}{l}\text { Correlación de } \\
\text { Spearman }\end{array}$ & 0,189 & 0,051 & 3,781 & $0,000^{\mathrm{c}}$ \\
\hline
\end{tabular}




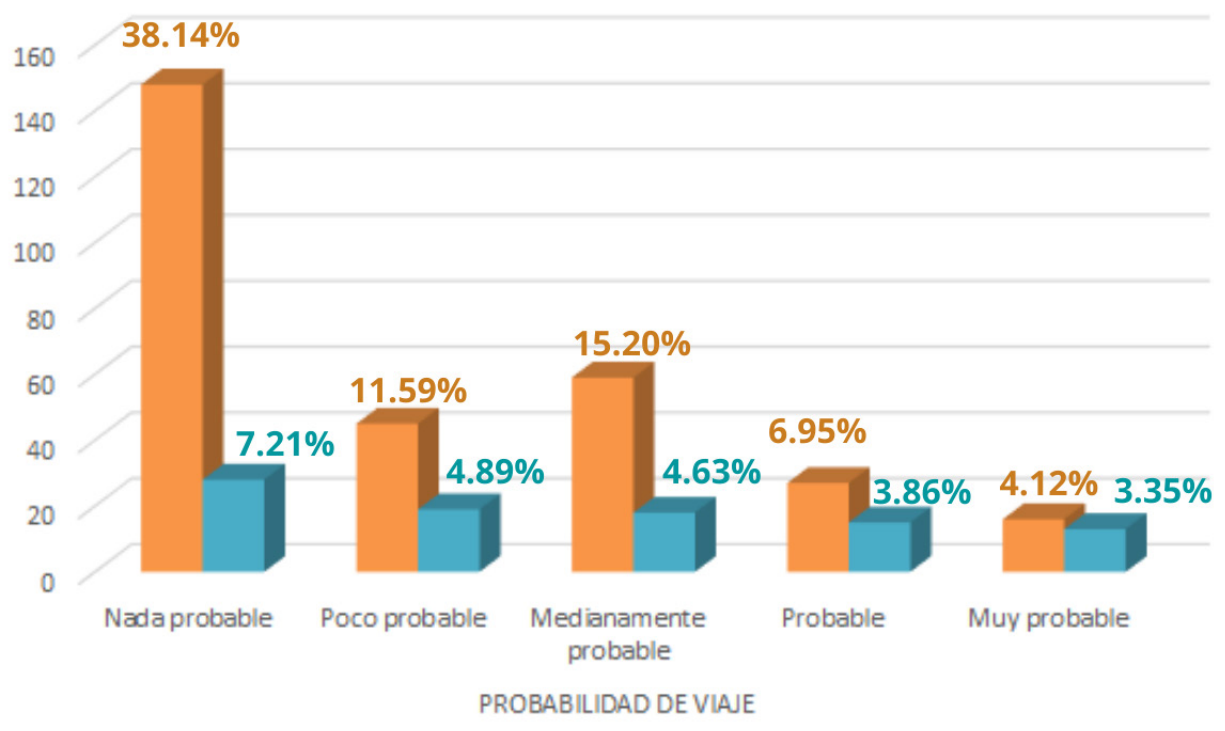

- REDUCIR EL NÚMERO DE VUELOS POR DÍA (ARRIBOS Y SALIDAS) Sí

- REDUCIR EL NÚMERO DE VUELOS POR DíA (ARRIBOS Y SALIDAS) No

Figura 8. Probabilidad de viaje vs. reducir el número de vuelos por día (arribos y salidas).

Para la cuarta y última correlación se tomó en cuenta 2 variables:

- Pregunta 3: Género de la persona encuestada.

- $\quad$ Pregunta 18: ¿Estaría usted dispuesto a pagar un valor extra para que la aerolínea en la que viaje le brinde implementos de seguridad sanitaria como son: gel antibacterial, mascarilla, guantes, traje de bioseguridad?

Se plantearon 2 hipótesis, una nula y una alternativa:

- $\quad \mathrm{H}_{0}$ : La compra de los implementos de seguridad con un valor extra en el ticket es independiente del género de los viajeros

- $\quad \mathrm{H}_{1}$ : La compra de los implementos de seguridad con un valor extra en el ticket es dependiente del género de los viajeros

La significación de la prueba de chi-cuadrado de Pearson $(0,035)$ (Tabla 7) y del coeficiente de correlación de Spearman $(-0,137)$ (Tabla 8) toma valores inferiores al nivel de significación del 0,05 considerado en este estudio. Esto indica que el es- tado de las variables analizadas es dependiente y la correlación entre ellas es débil, existe una dependencia entre la compra de los implementos de seguridad con un valor extra en el ticket y el género de los viajeros. Los resultados arrojan que un 55,55\% de hombres muestran un interés más alto en pagar un valor extra por la adquisición de implementos de seguridad, mientras que un 55,23\% de las mujeres no aceptarían pagar un valor extra por implementos de seguridad en el vuelo, para la obtención de estos porcentajes se realizó una sumatoria de los resultados obtenidos de la Figura 9. Estos resultados se encuentran relacionados con la conducta y psicología de hombres y mujeres que en su mayoría se enfocan en el comportamiento de la mujer al momento de conducir, ya que según varios estudios, uno de ellos Shinar et al. (2001), se menciona que las mujeres en comparación con los hombres manifiestan un comportamiento más prudente al volante, además cumplen en su mayoría con los 3 lineamientos de seguridad durante la conducción (uso del cinturón de seguridad, obedecer límites de velocidad y abstenerse al consumo de bebidas alcohólicas). Por lo tanto, se concluye que las mujeres son más precavidas en ciertos aspectos, uno de ellos, al momento de viajar en avión durante la pandemia COVID-19. 
Tabla 7. Pruebas de chi-cuadrado - correlación 4

\begin{tabular}{lccc}
\hline & Valor & Df & Significación asintótica (bilateral) \\
\hline Chi-cuadrado de Pearson & $4,459^{\mathrm{a}}$ & 1 & 0.035 \\
\hline
\end{tabular}

Tabla 8. Medidas simétricas - correlación 3

\begin{tabular}{llcccc}
\hline & Valor & $\begin{array}{c}\text { Error estándar } \\
\text { asintótico }\end{array}$ & T aproximada & $\begin{array}{c}\text { Significación } \\
\text { aproximada }\end{array}$ \\
\hline $\begin{array}{l}\text { Intervalo por } \\
\text { intervalo }\end{array}$ & R de Pearson & $-0,107$ & 0,050 & $-2,118$ & $0,035^{\mathrm{c}}$ \\
$\begin{array}{l}\text { Ordinal por } \\
\text { ordinal }\end{array}$ & $\begin{array}{l}\text { Correlación de } \\
\text { Spearman }\end{array}$ & $-0,107$ & 0,050 & $-2,118$ & $0,035^{\mathrm{c}}$ \\
\hline
\end{tabular}

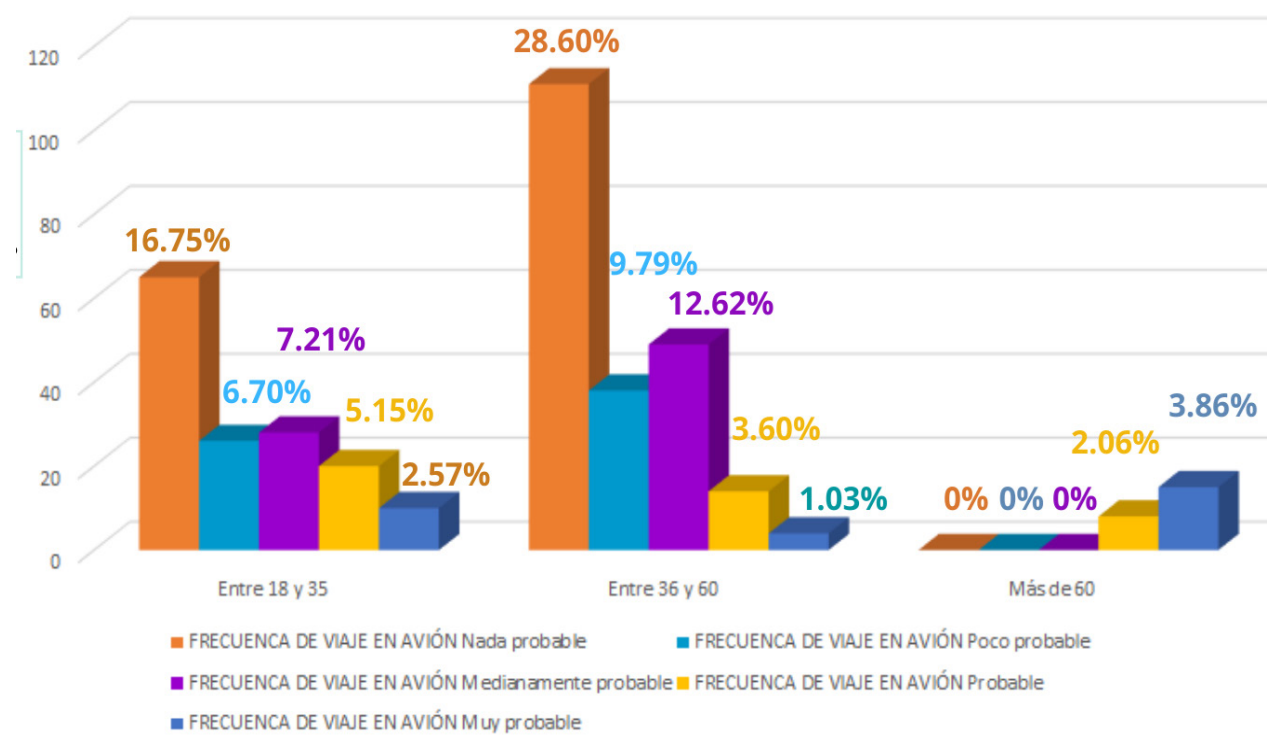

Figura 9. Pagar extra \& género.

\section{Discusión}

Siendo los viajeros la mayor fuente de ingreso de la aviación y del turismo, estos han tenido un cambio abrupto de perspectiva y necesidades, estas pueden variar dependiendo de ciertos factores. Actualmente, la prioridad de los clientes que utilizan el transporte aéreo, es la seguridad sanitaria que les puedan ofrecer en aeropuertos, aerolíneas y destinos. Las características pueden variar en género, edad, gustos y preferencias.

El análisis e interpretación de los resultados de los datos individuales de la encuesta y de las correlaciones, permitió crear tres distintos tipos de perfiles con relación al grupo etario de los encuestados. El primer perfil es de hombres y mujeres con un rango de edad entre 18 y 35 años con las siguientes características:
- Se encuentran más seguros al viajar con la mampara acrílica (resultado basado en la pregunta 11 de la encuesta).

- Están dispuestos a pagar un valor extra para que la aerolínea les brinde implementos de seguridad sanitaria (resultado basado en la pregunta 18 de la encuesta).

- Se encuentran totalmente de acuerdo en la reducción del número de visitas a pasajeros en arribos y salidas de vuelos (resultado basado en la pregunta 14 de la encuesta).

El segundo perfil es el de hombres y mujeres con un rango de edad entre 36 y 60 años con las siguientes características: 
- No están dispuestos a pagar un valor extra para que la aerolínea les brinde implementos de seguridad sanitaria (resultado basado en la pregunta 18 de la encuesta).

- Están de acuerdo con la reducción del número de personas que ingresen al aeropuerto para recibir o despedir a pasajeros (resultado basado en la pregunta 14 de la encuesta).

- Y se sentirían más seguros al viajar con mampara acrílica (resultado basado en la pregunta 11 de la encuesta).

El tercer y último perfil es el de hombres y mujeres mayores de 60 años de edad, con las siguientes características:

- Están totalmente de acuerdo con la reducción del número de personas que ingresen al aeropuerto para recibir o despedir a pasajeros (resultado basado en la pregunta 14 de la encuesta).

- Se encuentran más seguros al viajar con el asiento de la mitad en posición inversa (resultado basado en la pregunta 11 de la encuesta).

- Se encuentran dispuestos a pagar un valor extra para que la aerolínea les brinde implementos de seguridad sanitaria (resultado basado en la pregunta 18 de la encuesta).

No obstante, existen características dentro de la perspectiva del consumidor de transporte aéreo, que tienen una gran similitud; por ejemplo, sin importar las variables que diferencian a los viajeros, todos tienen algunas preferencias iguales. Se encuentran dispuestos a ser examinados en el check-in previo a su viaje, se encuentran cómodos al pasar por un filtro de sanidad previo al abordaje del avión y están de acuerdo con la reducción de visitas en las plataformas de arribo y salidas de los aeropuertos.

Existen ciertos antecedentes que influyen en estos resultados y todos están ligados con los autores que se mencionaron con anterioridad. El compor- tamiento de los viajeros cambió debido a distintos factores que forman parte de la llamada "nueva normalidad", se evidencia que en investigaciones anteriores relacionadas al comportamiento sociológico y psicológico de la población, en general, no existe un cambio total de la conducta del consumidor, pero sí hay una modificación y adaptación en su percepción, la cual se vincula a los nuevos comportamientos de los pasajeros que consumen el servicio de transporte aéreo, en este caso, en la ciudad de Quito. Por lo tanto, es posible que las nuevas conductas se mantengan en la psicología natural del ser humano, ya que, actualmente la percepción en la seguridad sanitaria es vista de forma parcial.

\section{Conclusiones}

Esta investigación ha visto la necesidad de marcar un precedente en la demanda para el consumo de transporte aéreo. El cambio en el razonamiento y perspectiva del consumidor es muy evidente, por lo cual, esta investigación ayudará a la creación de planes de adaptación, protocolos de bioseguridad y, sobre todo, un servicio innovador que tenga como prioridad la seguridad sanitaria para generar confianza en el consumidor de transporte aéreo.

Además, se planteó una metodología de estudio del comportamiento del consumidor quiteño de este tipo de servicio, puesto que no se han realizado estudios como este en el pasado y ha sido de gran ayuda para entender de mejor manera las necesidades del consumidor actual, puesto que a raíz de la pandemia se ha priorizado la seguridad sanitaria antes que otros factores.

En un futuro, se podrían realizar más estudios de este tipo utilizando esta metodología, tomando en cuenta otras variables que influyen de manera directa en el comportamiento del consumidor, tales como: factores culturales y tecnológicos, motivación de viaje, segmentación y la percepción del servicio de transporte aéreo, incluso se podría realizar otro estudio similar a este, pero tomando en cuenta las nuevas medidas que ha adoptado el aeropuerto de la capital.

\section{Referencias}

Aeropuerto Internacional de Quito. (2020). Protocolos y medidas sanitarias empleados en el Aeropuerto Internacional Mariscal Su-

cre de Quito. https://www.aeropuertoquito. aero/es/protocolo-covid-19.html 
Aviointeriors. (2020). Glassafe \& Janus. http:// aviointeriors.it/prodotti/glassafe/

Martínez Bencardino, C. (2012). Estadística y muestreo (13 $\left.{ }^{\mathrm{a}} \mathrm{ed}.\right)$. Ecoe Ediciones.

Hernández Sampieri, R., Fernández Collado, C., \& Baptista Lucio, P. (2013). Metodología de la Investigación (5 ed.). McGraw-Hill Interamericana.

IBERIA. (2020). Medidas adoptadas por el COVID-19. https://www.iberia.com/ec/es/covid-19/medidas-adoptadas/\#: :text=pueden $\% 20$ estar $\% 20$ cerrados.-,En\%20el\%20aeropuerto,cubran $\% 20$ nariz $\% 20 \mathrm{y} \% 20$ boca $\% 20$ siempre

Larenas, N. (2020). IATA: grandes pérdidas para la industria aérea durante el 2020. https://www. nlarenas.com/2020/06/iata-perdidas-industria-aerea-durante-2020/

Meulman, J. J., \& Heiser, W. J. (2005). Categories 14.0. SPSS Inc.

Ministerio de Salud y Protección Social de Colombia. [MinSalud]. (2020). El riesgo de contagio de covid-19 en los aviones es mínimo. https:// www.minsalud.gov.co/Paginas/El-riesgo-decovid-19-en-los-aviones-es-minimo.aspx

Ministerio de Turismo [MINTUR]. (2020a). Llegadas y Salidas Internacionales en Ecuador. https://servicios.turismo.gob.ec/index.php/turismo-cifras/2018-09-19-17-01-51/movimientos-internacionales

Ministerio de Turismo [MINTUR]. (2020b). Ecuador recibe un sello internacional por ser un destino seguro para los viajeros. https://www. turismo.gob.ec/ecuador-recibe-un-sello-internacional-por-ser-un-destino-seguro-para-los-viajeros/

Murello, D. (2017). Los adultos mayores como consumidores de turismo. Caso de estudio: Ciudad de Bahía Blanca. Universidad Nacional de Quilmes.

Organización Internacional del Trabajo [OIT]. (2020). Observatorio de la OIT: La COVID-19 $y$ el mundo del trabajo. $5^{a}$ ed. Estimaciones actualizadas y análisis. https://www.ilo.org/ wcmsp5/groups/public/@dgreports/@dcomm/ documents/briefingnote/wcms_749470.pdf
Organización Mundial de la Salud [OMS]. (2020a). Preguntas y respuestas sobre la transmisión de la COVID-19. https://www.who. int/es/news-room/q-a-detail/q-a-how-is-covid-19-transmitted? gclid $=\mathrm{CjwKCAjwkJj-}$ 6BRA-EiwA0ZVPV15FRu7NEI1 haxTYc-ohqOi157bbGWIzI6HENyHRLU_qqK4V3BAbZRoClp8QAvD_BwE

Organización Mundial de la Salud [OMS]. (2020b). Vacunas e inmunización: ¿qué es la vacunación? https://www.who.int/es/newsroom/q-a-detail/vaccines-and-immunization-what-is-vaccination? adgroupsurvey $=\{\mathrm{ad}-$ groupsurvey $\} \&$ gclid=Cj0KCQiA3NX_BRDQARIsALA3fILhihTQebe-K-o9vg1CWgjpHpFGpSQ1aV8hPcxMxRKkEm00sXF-prQaAseHEALw_wcB

Secretaría Nacional de Información [SNI]. (2017). Proyecciones y estudios demográficos. https://sni. gob.ec/proyecciones-y-estudios-demograficos

Shinar, D., Schechtman, E., \& Compton, R. (2001). Self-reports of safe driving behaviors in relationship to sex, age, education and income in the US adult driving population. Accident; analysis and prevention, 33(1), 111-116. https://doi.org/10.1016/s0001-4575(00)00021-x

SPSS [Statistical Product and Service Solutions]. Versión (20). IBM.

López, A. M. (2020). Economic impact of coronavirus (COVID-19) on the tourism sector in Ecuador in 2020, by scenario (in million U.S. dollars). Statista. https://www.statista.com/ statistics/1106008/coronavirus-economic-impact-tourism-scenario-ecuador/

Suau-Sanchez, P., Voltes-Dorta, A., \& Cugueró-Escofet, N. (2020). An early assessment of the impact of COVID-19 on air transport: Just another crisis or the end of aviation as we know it? Journal of Transport Geography, 86, 102749. https://doi.org/10.1016/j.jtrangeo.2020.102749

Subía, M. (2020). Entrevista realizada por Fabricio Cevallos en Ecuador Tv. https://www. ecuadortv.ec/categoria/telediario/noticias/aereo-sector-industria

The College of Physicians of Phyladelphia. (2018). Desarrollo, pruebas y reglamentos para las va- 
cunas. https://www.historyofvaccines.org/es/ contenido/articulos/desarrollo-pruebas-y-reglamentos-para-las-vacunas

Whitehead, J. (2020). Invasion of the Body Snatchers: Psychological warfare disguised as a pandemic threat. The Ricchmond
Observer. https://www.richmondobserver. com/opinion/item/10470-opinion-invasionof-the-body-snatchers-psychologicalwarfare-disguised-as-a-pandemicthreat.html? fbclid=IwAR33uU9FRvq O k n D P D H Q 0 b q w 2 w z K oH2AHIIJNLfC8ZSWpDspLz8DCfiePc 